Journal of Computer Science 6 (3): 312-319, 2010

ISSN 1549-3636

(C) 2010 Science Publications

\title{
Nodes Density and Broadcast Management in Heterogeneous Environments of Mobile Ad-Hoc Networks
}

\author{
Essam Natsheh and Khalid Buragga \\ Department of Information Systems, College of Computer Sciences and Information Technology, \\ King Faisal University, P. O. Box (55130), Al-Hassa 31982, Saudi Arabia
}

\begin{abstract}
Problem statement: The research presented in this study looks into Mobile Ad-Hoc Networks (MANETs) environment that has varying node densities, called heterogeneous density environment. Such environment can be roughly associated with situations varying from areas struck by disasters to normal city environments. Approach: This study improved the performance of existing MANETs routing protocols by reducing the communication overhead incurred during the route discovery process. This reduction in communication overhead is achieved by implementing a new broadcast protocol. The proposed broadcast protocol is based on the density and connectivity of the nodes and not just the number of nodes. It compared against the will known routing protocols using simulation. The simulation is conducted in three different environments: dense environment, varying dense environment, and sparse dense environment. Results: Extensive performance analysis proves the effectiveness of the proposed protocol in terms of packet delivery ratio and throughput in the three environments. In dense environments the increase in performance is obvious for packet deliver ratio which in the end translates into high throughput. The throughput is seen to be improved from the original protocol by at least $10 \%$ and this trend is also observed in other forms of environment. Conclusion: The performance of the proposed protocol has been encouraging by the fact that in the three different densities environments it has shown that there is a conspicuous increase in performance for packet delivery ratio and throughput. This illustrated the effectiveness of the proposed protocol in bandwidth utilization for data transfer in different environments.
\end{abstract}

Key words: Ad-hoc networks, nodes density, broadcast management, routing protocols

\section{INTRODUCTION}

Mobile Ad hoc Networks (MANETs) are basically non-infrastructure based networks with an undefined network size. This is due to the ubiquitous nature of the MANETs that allows any device to be attached to a certain network anytime. It is only limited by range of the wireless transmission. Thus, there are many problems and issues that need to be addressed for MANETs protocols. One of the main issues is the nodes movement and the dynamic change that occurs in the network topology.

This study proposed on studying the operation of MANET in an environment with varying network node densities, called heterogeneous density environment. Previous studies on the impact of MANET node density have shown that MANET operation is very dependent on the availability of neighbor nodes. Royer et al. (2001) have shown that MANET nodes would either have to move at moderate speeds or increase its transmission power in order not to be isolated from the network. Otherwise, a sparsely populated MANET environment would suffer a significant performance drop or would not be connected at all. Mobility for MANET nodes is one of the main problems identified when MANET routing is performed. Nodes with constant motion are expected to form temporary groups or sub-network within the original MANET. Thus, when these nodes travel at high speeds the topology of the MANET becomes even more inconsistent and consequently degrading its network performance.

Deploying MANET in urbanized areas with a mixture of vehicular and pedestrian traffic and disaster areas would fit into the scenario where node densities vary from time to time. The main contributing factor would be node mobility creating small non-uniform networks within the same network area.

To overcome the above problem, the routing protocol broadcast the routing information via the network. That consequently issues a problem known as

Corresponding Author: Essam Natsheh, Department of Information Systems, College of Computer Sciences and Information Technology, King Faisal University, P. O. Box (55130), Al-Hassa 31982, Saudi Arabia 
"broadcast storm". This event occurs when a high number of broadcast activities are performed simultaneously at a certain period of time and triggering torrents of redundant broadcasts requests and replies that will eventually lead the contention based link layer of MANETs to suffer a blackout (Ni et al., 1999). In networks with varying node densities, such problems is expected to occur more frequently as MANET nodes will be forced to reiterate its broadcasting events whenever there is a broken link or when the destination could not be found over a certain period of time. The performance of the communication in the network will then eventually decline over time.

In short, the problems identified in this study on MANETs with varying node densities are: low packet delivery, low throughput, high end to end delay and potential "broadcast storm" problems due to unmanaged network broadcasting soliciting very high number of routing overheads in highly dense areas of the network.

The proposition to solve the problems discussed earlier will be a density based probabilistic algorithm to be implemented on MANET routing protocols. The proposed algorithm will be neighbor aware and is expected to perform broadcast at rate which is determined by the density of the network. The algorithm is expected to collect neighbor information based on an incremental counter for every route update that is performed prior to performing the Route Discovery process. Thus, the algorithm is expected to perform less frequent broadcasting activities when there are a high number of neighbors around and will assume normal broadcasting activities when the amount of neighbors are low. The objective of the algorithm is to lower the amount of routing packets per data packet as to increase the efficiency of the network. It also limits the number of broadcast reiterations performed based on the density of the nodes in the same area. The number of broadcast iterations in the current implementation is not adjusted according to the topology of the network. The situation increases the possibility of soliciting a torrent of route replies from other nodes receiving the broadcast. The replies would then contribute to the "broadcast storm" problem. While reducing the number of broadcast iterations according to the density of the network will help dense networks to reduce the amount of packet collisions that may occur due to a "broadcast storm". On the other hand, sparse networks would continue to perform its broadcasting activities similar to the current implementations.

Common MANETs' routing protocols: A few of the popular routing algorithms for MANETs would include distance vector algorithms, hierarchical, link state and also source routing algorithms. Most notable Proactive routing algorithms using distance vectors are Destination Sequence Distant Vector (DSDV) protocol and Reactive protocols which uses distance vector would have to be Ad-hoc On-Demand Distance Vector (AODV) protocol. Distance vector algorithms require each node to maintain a set of distances which includes a set of neighbors over a certain range defined by the number of hops. Periodic updates on the neighbor distances allow an accurate approximation of the closest neighbor and location of the shortest path taken to the destination.

Other protocols that utilize link state algorithms are also often a subject of research in MANETs. Link State algorithms are used in Global State Routing (GSR), Fish Eye Routing (FSR) (Abolhasan et al., 2004) and Optimized Link State Routing (OLSR) (Jacquet et al., 2001). Link state approach to routing required nodes in the network to acquire the routing information of other nodes in the network via a periodic update on the information over a certain period of time. The information would usually be flooded over the entire network regardless of whether if there are active transmitting links. Upon receiving the link state algorithm a shortest path algorithm will be applied to node for selecting the route towards the destination.

Some MANETs routing algorithms are based on clustering where nodes are gathered into clusters in a network. Usually in algorithms like these a cluster head is elected to manage the other nodes in the cluster. Such algorithms can be seen in protocols such as Cluster-head Gateway Switch Routing (CGSR) (Abolhasan et al., 2004). The cluster head is expected to manage the transmissions of nodes that pass through it and other inter-cluster communications as well. The other nodes in the cluster would normally only maintain its communication to the cluster head only unlike other protocols which require all nodes in the network to store a significant amount of routing information.

Source Routing is also considered one of the more popular algorithms in MANETs routing as its experiments yielded very good results in reactive protocols (Johnson and Maltz, 1996). The most notable source routing protocol available in MANETs is the Dynamic Source Routing protocol (DSR). Source Routing requires the nodes in the network to listen to every message that is flooded into the network and store them as part of the routing information, regardless if the information is relevant to its current state. Whether if the node is transmitting or it remains idle any broadcast information will be intercepted, stored and forwarded. This provides each node in the entire 
MANETs with routing information of other nodes as well.

Other complex routing algorithms like geographic based protocols like Distance Routing Effect Algorithm for Mobility (Abolhasan et al., 2004) (DREAM) and Geography-aided Multicast Zone Routing Protocol (GMZRP) (Cheng et al., 2009) sometimes requires geographic coordination through a GPS. Geographic coordinates plays a vital role in determining the routing table and choice of route for the nodes. Scalability of the network is increased and the consumption of bandwidth is expected to be lower. Nodes that are confined in certain known areas would not have to update their routing information.

AD-hoc On-Demand Distance Vector (AODV) routing protocol: Ad-hoc on Demand Distance Vector (AODV) protocol (Perkins et al., 2003) is reactive adhoc wireless protocol that is able to support unicast, broadcast and multicast data transmission. AODV uses the next hop routing model with sequence numbers and periodic beacons to discover routes and maintain them. These features were borrowed from Destination Sequenced Distance Vector (DSDV) routing protocol (Perkins et al., 2003). AODV uses Route Discovery and Route Maintenance as basic mechanisms for establishing links among nodes.

AODV Route Discovery is required when the node has data to send. A link to the desired destination must be established before any upper layer communications could take place. Route Discovery begins with a route Request (RREQ) message containing information such as the destination node's IP address, sequence number, hop count and broadcast ID. These attributes are sufficient for identifying the destination node; therefore if a non destined node receives the message it will automatically forward it until the message reaches its intended recipient (Fig. 1). A route reply (RREP) will be sent back to the message source using the reverse route obtained from the RREQ message (Fig. 2), otherwise a new route has to be determined using the Route Discovery mechanism if no route exists.

Route Maintenance is required by AODV to ensure that the selected or discovered routes are fresh (current), up to the point where the sender is initiating upper layer communication. The freshness of the routes is recorded in the routing table entries maintained by each node. To reduce the amount of RREQs flooding the network in the event of any failed links, an optimization technique is introduced. The technique uses the Expanding Ring Search (Hasan and Jha, 2004) to locate other alternative paths to replace a failed link.

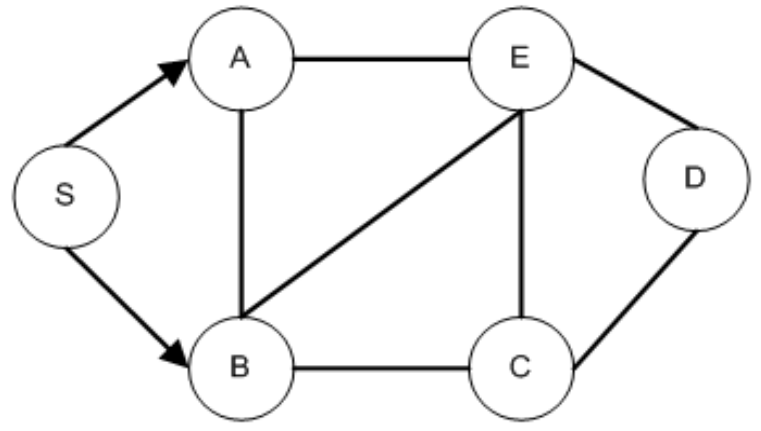

Fig. 1: A route request cycle for AODV where $S$ is the source and $\mathrm{D}$ is the destination

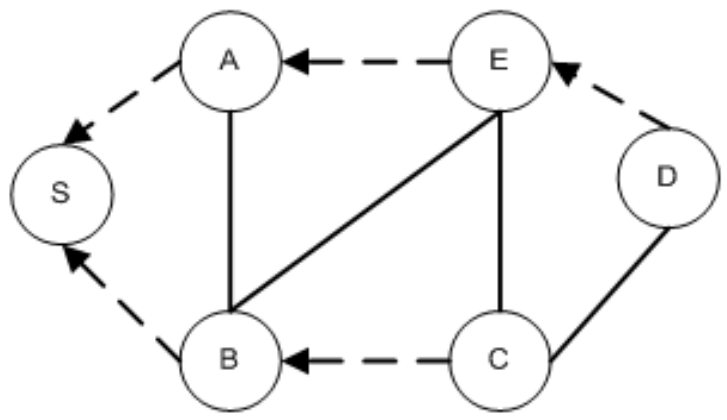

Fig. 2: A route reply is sent from the destination back to the source creating a reverse path

Comparison of MANETs' routing protocols: Comparison studies using metrics such as Packet Delivery Ratio, Throughput, Dropped Packets and Path Optimality have been conducted and evaluated against one another. The study by (Yongsheng et al., 2008) focuses on the comparison between Reactive and Proactive protocols. Four basic MANETs routing protocols were tested in similar environments. The analysis of the study, proactive protocols like DSDV did not measure up to the performance of its reactive counterparts like AODV and DSR. Several determining factors exhibited that Proactive protocols were not scalable enough and suffered when there is high mobility. The study concluded that reactive MANETs routing protocols perform better for networks that are more dynamic, with high node mobility. The protocols were evaluated in terms of packet delivery ratio, Average End to End Delay per packet and normalized routing overheads. Packet Delivery Ratio is the amount of packets successfully received by the destination node over the total number of packets sent throughout the entire simulation. Average End to End Delay per packet is the amount of time taken by a single packet to travel from source and successfully reaching the destination. 
Normalized Routing Overheads are the amount of routing packets over the over the number of packet successfully received at the destination.

In a study by Das et al. (2000), DSR and AODV were compared against each other. The author highlighted the usefulness of promiscuous listening which allows DSR to possess a great amount of routing information compared to what AODV might have in its routing table. Therefore AODV need to perform more route discoveries compared to DSR in a similar environment. On the other hand, AODV can almost always ensure that the selected route is fresher since the selection of routes is determined by the sequence number and based on the most recent routing entry. It was observed that more experiments should be conducted to examine the inter-layer coupling between the routing layer and the data link layer to provide a better insight into how both protocols work.

Another comparison study was made between AODV and OLSR (Huawei and Yun, 2008) in terms of scalability, security considerations and resource usage. The comparison was made via referencing the architecture of the two ad-hoc routing protocols and other literature that is concerned of the protocols either individually or compared to other protocols as well. The OLSR is deemed to performed better in networks with high density and a high frequency of data transmission. Other issues such as scalability are proven to be a challenge for both the routing protocols. Security however favors AODV as it is observed to be able to cope with more complex cryptographic solutions. The study illustrates an overall picture of how well the performances of the two routing protocols behave but more detail elements are neglected. The contribution from this literature provides an overview on the difference of proactive distance vector protocol versus reactive link state routing protocols.

Conclusively, to study the nodes density and broadcast management issues, an ad-hoc routing protocols will be selected. The selection of the ad-hoc routing protocol will be based on observation of previous studies mentioned before. The observation of the routing protocol would be based on its robustness of its performance in high density environments and high mobility scenarios. Therefore the AODV routing protocol was selected due to several reasons:

- Extensive studies were performed to evaluate the protocol's scalability

- Density issue on AODV protocol was also discussed

- It have been implemented in real implementations namely AODV-UU (2009)
- Other issues like security and handover issues were also well discussed in related studies

Nodes density issue: The optimum density of MANETs was studied in (Royer et al., 2001), which discussed the tradeoffs between network density and node connectivity in the face of increasing node mobility and proposed an optimal node density for maintaining connectivity in a stationary network. However, the results were inconclusive regarding the optimal density for maintaining connectivity in highly mobile environments. When neighbor nodes are saturated they yield almost similar results. For instance the number of nodes converge around 3 and the throughput converged around less than 0.1 . Nonetheless, (Royer et al., 2001) concluded that both transmission power and the node densities need to increase when nodes experience increasing mobility if connectivity were to be maintained.

In real life situations large mobile networks will not have uniform sets of nodes populating the network area. Instead, groups of nodes varying in numbers would be found scattered around the network area. This situation can be reflected in the normal urban mobile network environment setup or other scenarios such as disaster areas and vehicular networks. Therefore to evaluate the performance of MANETs in an environment without considering the situations mentioned earlier could not provide a clear picture on the scalability of the MANETs routing protocols.

Network node density for an entire network can be differentiated into physical density versus connectivity density. In this study network physical density is defined as dense when large number nodes are in proximity of one another within a particular area and vice versa for sparse. However, when determining density for a particular network, one should also consider the connectivity of the network in terms of transmission range that covers the particular area. Thus the network density determined in this study, is based on the number of nodes found in a particular area and the connectivity of the nodes. Therefore even though the number of nodes found in a small area may not be packed, given a high transmission range then it can be determined that the node in the area is dense. Otherwise given either a very large or low connectivity the node density could be determined as sparse.

On the issue of connectivity density, studies by (Bettstetter, 2002; Bettstetter and Zangl, 2002) discussed determining the network connectivity based on the density of the numbers of neighboring nodes. The density is defined based on the transmission range 
of the nodes. The definition of connectivity density of a network based upon the study is as follow:

- The number of neighbors surrounding a node is denoted by its degree $d$

- A node that has a degree $\mathrm{d}=0$ is said to be isolated from the rest of the network

- The minimum degree of nodes $\mathrm{d}_{\min }$ and is considered as the smallest degree of all nodes in the network

- A network is said to be connected when every pair of nodes exists a path between them, otherwise it is disconnected

- A connected network always has a minimum degree $d_{\text {min }}>0$ but the reverse implication is not necessarily true

- A network is k-connected if for each pair of node exists $\mathrm{k}$ mutually independent paths connecting them

$\mathrm{P}$ is the probability of the connectivity. The value $\mathrm{n}$ is the number of nodes located in the area. The value $\mu$ is represented by Eq. 2 where $\rho$ is the density, $\pi$ represents the circumference and $r$ is the radius of the transmission:

$\mathrm{P}(\mathrm{k}-$ con $) \approx\left(1-\mathrm{e}^{-\mu}\right)^{\mathrm{n}}$

$\mu=\rho \times \pi \times r_{0}^{2}$

$\rho=n / A$

Based on this one can have the criteria for determining the size of each 'square' in the topology. In this study the value of $\mathrm{k}$ is set to1. This means that in any particular network mentioned as dense given the probability of the connection of $\mathrm{P}(\mathrm{k}-\mathrm{con}) \geq 0.95$ where $\mathrm{k}=1$, there is 1 mutually independent path connecting the nodes in the particular network area. Thus the network is categorized as (almost surely) 1-connected. This also implies that for any neighbors found within the transmission range of a particular node they are at most 2 hops away from each other. The scenario can be best described based on the illustration shown in Fig. 3.

The node density of the network areas in this study will be based on the formulae provided for $\mathrm{P}(\mathrm{k}-\mathrm{con})$. Therefore an area is considered dense when a MANET source node identifies that:

- It neighbors are at most 2 hops away from it and it has a mutually exclusive path to other neighboring nodes that is independent of one another

- $\mathrm{P}(1$-con $) \geq 0.95$
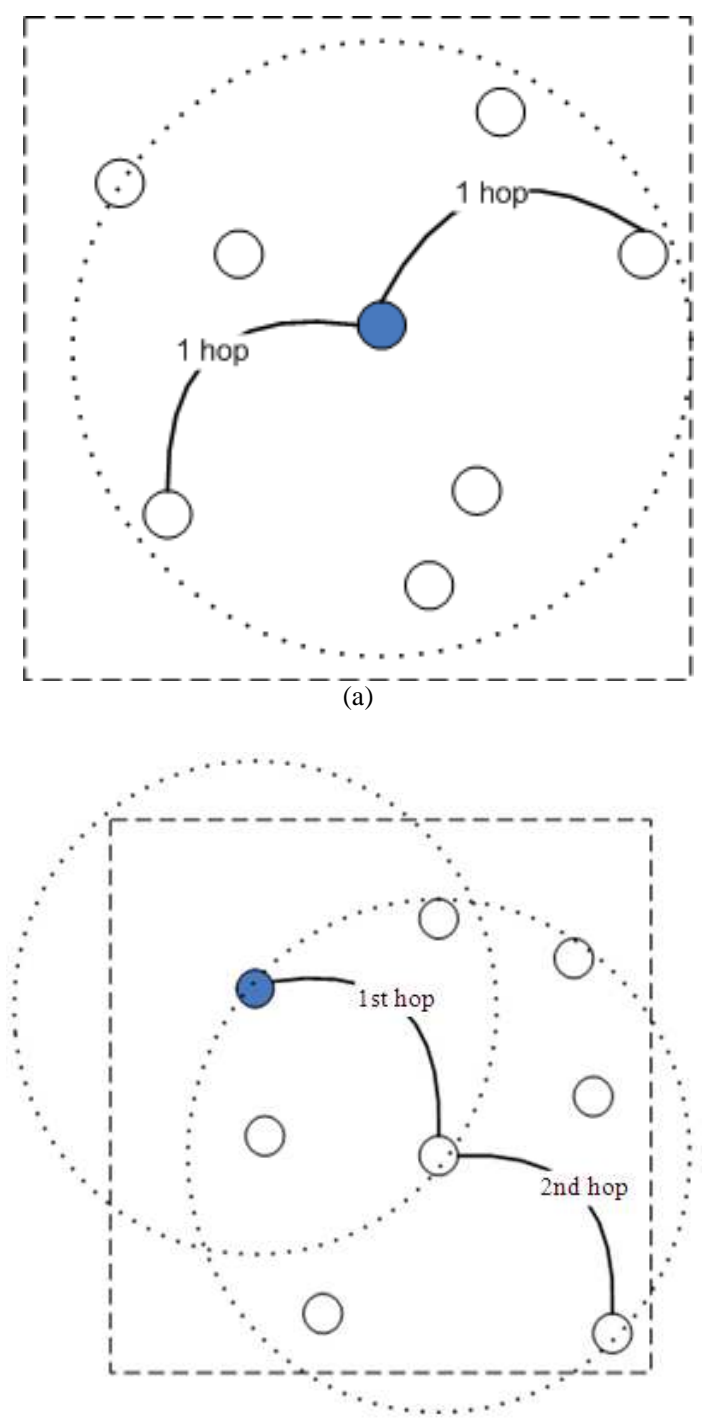

(b)

Fig. 3: Conditions of the node connecting to its neighbors with 1 located at the center of the network area and the other stuck on one side of the network. (a) Source located in the center of the area: (b) Source not located at the center of the area

Sparse areas will be areas where nodes are isolated from a network or from one another:

- Nodes in a sparse neighbor cannot guarantee at least a single connection in the network (P (1-con) $\leq 0.95$ )

- The minimal neighbor node degree for sparse areas could be $d_{\min }=0$. Thus, the node could be disconnected from the network 
To alleviate certain known problems in MANETs many alterations to the original routing protocols have been introduced. Some required small and some required an overhaul of the original algorithms. In many cases MANETs routing protocols alterations relates to the applications in which it is intended for. There are also intentions to adopt MANET technology in vehicular networks and some in sensor networks as well. Whatever the intention the MANETs is design for some common or basic problems still remains in the available protocols when it is moved to into real implementations.

Broadcast management issue: The nature of our proposed scheme is a combination of the issues of nodes connectivity (density) and broadcast management. We'll conduct a survey on broadcast management issue and then we'll propose the new route discovery algorithm.

Literature review on broadcast management in MANETs: Flooding based broadcast activities in MANETs are one of the main contributing factors to the drop in network performance. As highlighted in (Ni et al., 1999) broadcast activities in MANETs are both unreliable and spontaneous. For on-demand MANETs routing protocols the problems caused by unmanaged broadcast activities worsen, as broadcast based Route Discovery are performed for every unknown route in the network. The study also pointed out that flooding based broadcast creates redundant broadcasts, network contention and frequent packet collisions. The identified problems were further verified by probability calculations and several schemes were proposed to alleviate the problems. The schemes were counter-based schemes, probabilistic schemes, location aided, distance schemes and location aided schemes.

An analysis on probabilistic broadcast for MANETs was conducted in $(\mathrm{Wu}$ and Lou, 2003; Stojmenovic et al., 2002; Tseng et al., 2003; Colagrosso, 2007). These studies analyze MANETs' performance with adjusted probability for flooding. The analysis looked into issues such as saved rebroadcasts, reachability, mobility and node density. Saved rebroadcasts are the number of redundant broadcasts activities that are prevented or stopped. The observation from these studies found that low mobility contributes to more saved rebroadcasts. The different probability values used in different types of speed and node density affects the reachablity and saved rebroadcasts. Thus, these studies prompt for a dynamic probabilistic scheme to be introduced to cope with the varying types of MANETs' environments.
Dynamic probabilistic broadcast (Zhang and Agrawal, 2005) was introduced to reduce the amount of flooding performed on MANETs by performing flooding via a probabilistic based broadcast based on the packet ID information. The packet ID is stored in an array list, where each redundant broadcast packet ID is incremented, while the more redundant packet is received the less probable that a broadcast is to be performed. The introduced scheme managed to reduced the amount of latency and generate fewer rebroadcasts compared to the fixed probability approach and counter based approach. The conclusion of the study showed that with a dynamic probabilistic scheme, MANETs routing protocols would have better management on flooding based broadcasts.

In (Siddique et al., 2007), the authors utilize the neighbor cache information for the AODV protocol that periodically updates it "active" neighbors for its node. The scheme introduced in the study utilizes a dynamic probabilistic broadcast coupled with the neighbor information. Thus the broadcast probability is based on the number of nodes that is kept in the neighbor cache. The scheme however does not determine whether if the neighbors in the network is proportion to the size of the network and it is does not tell of the algorithms performance against an inconsistent topology in terms of neighbor size and mobility.

With the broadcasting methods described above, they reduce the number of rebroadcasts at the expense of reachability, longer delay, need support from GPS and other location devices, or require the exchange of neighborhood information with hosts. In this study, we propose a new probabilistic approach that dynamically fine-tunes the rebroadcast probability for Route Discovery sending algorithm according to the density and connectivity of the neighbor nodes to yield higher throughput, higher saved rebroadcast, better reachability and lower rout request.

\section{MATERIALS AND METHODS}

For broadcast management in heterogeneous density environment, we suggest to change AODV's Route Discovery sending algorithm to be as shown in Fig. 4.

As illustrated in Fig. 5 the neighbor nodes will be decided by those that had recently replied the source node with a HELLO packet. The reply from the HELLO packet will be added into the cache until the next timeout occurs. The reply from each unique HELLO packet will be taken into account as neighboring nodes to determine the density. 


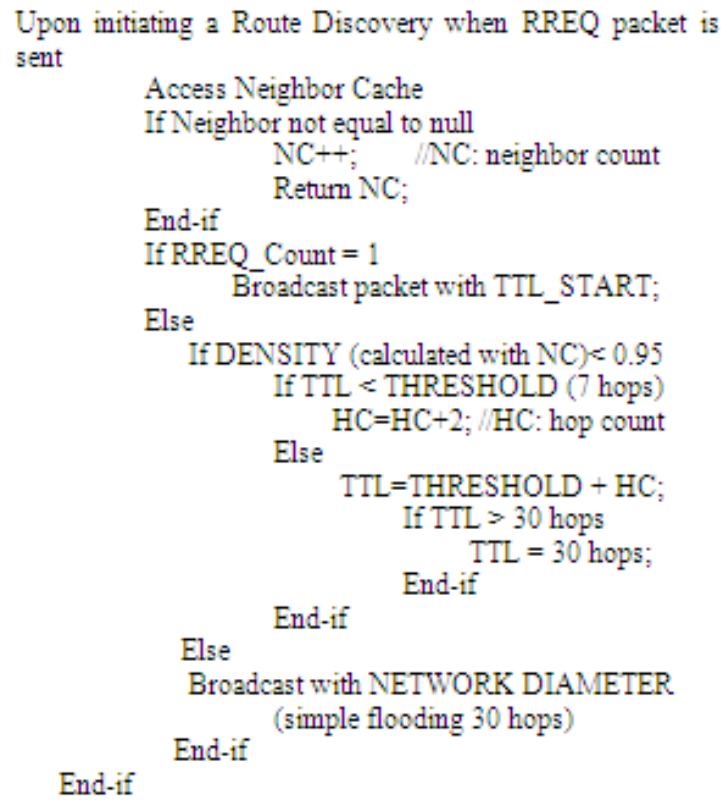

Fig. 4: Proposed AODV's route discovery sending algorithm

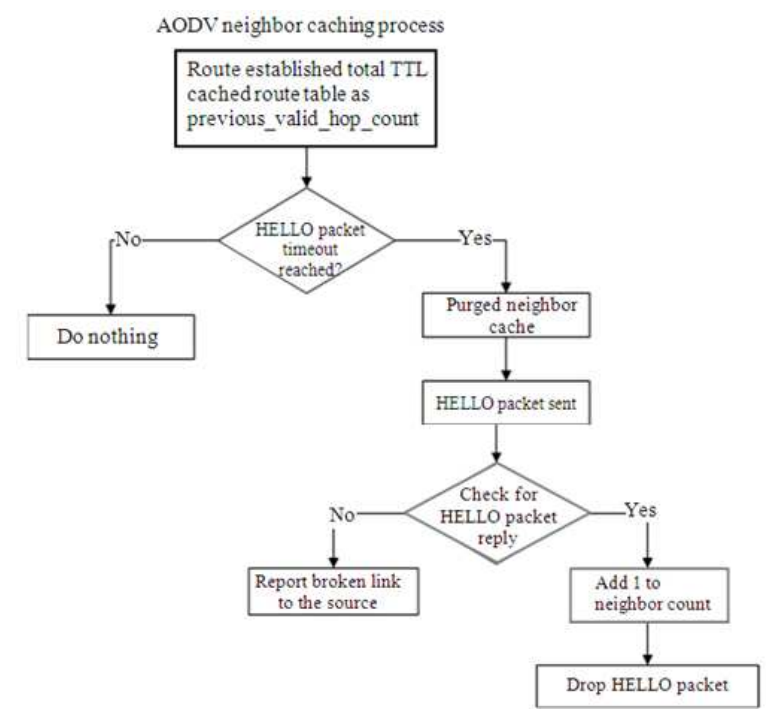

Fig. 5: Neighbor count acquisition mechanism for AODV

The number of the neighbors will change according to the mobility of the network thus for each neighbor cache purged the number of neighbors will reset and the process continues until the AODV protocol stops.

\section{RESULTS AND DISCUSSION}

The proposed algorithm will mostly depend on the density of the neighboring nodes to decide it next course of actions unlike the original AODV protocol. This aids the nodes in sparse areas to increase their ability to reach further when sending the RREQ message. The nodes in the dense areas will only extend its reach based on the THRESHOLD + HC to the destination after successive retries which the TTL is below 7. This is different from the original AODV where the entire network will be flooded at this point.

\section{CONCLUSION}

The literature review in this study gives an overview on environments with different node densities and has highlighted some issues which were previously not observed in normal environments. The introduction of MANETs in the early parts of this study explains the nature of MANETs and subsequently discussed on the most popular choice of MANETs routing protocol which is AODV. Then a review on the comparison for MANETs routing protocols was performed. The idea for the heterogeneous topology was discussed in detailed whereby, dense and sparse networks were determined. The final part of this study reviews the flooding based broadcast problems and certain measures to counter it. A scheme proposed in this part utilizes the probability of $\mathrm{P}(1-\mathrm{con})$ to determine the density of the network thus the broadcast probability is based on the density and connectivity and not just the number of nodes.

As a prospect for future work, we plan to evaluate the performance of adjusted probabilistic flooding on the AODV algorithm. Then we aim to build an analytic model for our approach in order to facilitate the exploration of the optimal adaptation strategy, with regard to probability setting and network density. Finally, since the technique avails itself to various types of network-wide dissemination, we plan to integrate it with a proactive routing protocol, namely OLSR. This protocol already incorporates techniques which reduce the effect of flooding and are orthogonal to our scheme, which implies the opportunity to examine if cumulative improvements with our method are possible.

\section{ACKNOWLEDGMENT}

The researchers would like to express their appreciation to Deanship of Scientific Research at King Faisal University for supporting this research.

\section{REFERENCES}

Abolhasan, M., T. Wysocki and E. Dutkiewicz, 2004. A review of routing protocols for mobile ad hoc networks. Ad Hoc Networks, 2: 1-22. DOI: 10.1016/S1570-8705(03)00043-X 
AODV-UU, 2009. Communication research. MS Thesis. http://www.it.uu.se/research/group/coregroup/

Bettstetter, C. and J. Zangl, 2002. How to achieve a connected ad hoc network with homogeneous range assignment: An analytical study with consideration of border effects. Proceeding of the IEEE International Conference on Mobile and Wireless Communication Networks (MWCN'02), IEEE Xplore Press, Stockholm, Sweden, pp: 125-129. DOI: 10.1109/MWCN.2002.1045708

Bettstetter, C., 2002. On the Connectivity of wireless multihop networks with homogeneous and inhomogeneous range assignment. Proceedings of the IEEE 56th Vehicular Technology Conference, (VTc'02), IEEE Xplore Press, Vancouver, BC., Canada, pp: 1706-1710. DOI: 10.1109/VETECF.2002.1040507

Cheng, H., J. Cao, and X. Fan, 2009. GMZRP: Geography-aided multicast zone routing protocol in mobile ad hoc networks. Mobile Networks Appli., 14: 165-177. DOI: 10.1007/s11036-0080135-4

Colagrosso, M.D., 2007. Intelligent broadcasting in mobile ad hoc networks: Three classes of adaptive protocols. EURASIP J. Wireless Comm. Network, 2007: 25-40. DOI: 10.1155/2007/10216

Das, S.R., C.E. Perkins and E.M. Royer, 2000. Performance comparison of two on-demand routing protocols for ad hoc networks. Proceedings of the IEEE 19th Annual Joint Conference of the IEEE Computer and Communications Societies, (CCS'00), IEEE Xplore Press, Tel Aviv, pp: 3-12. DOI: $10.1109 /$ INFCOM.2000.832168

Hasan, J., and S. Jha, 2004. Optimizing expanding ring search for multi-hop wireless networks. Global Telecommun. Conf., 2: 1061-1065. DOI: 10.1109/GLOCOM.2004.1378120

Huawei, Z. and Z. Yun, 2008. Comparison and analysis AODV and OLSR routing protocols in ad hoc network. Proceedings of the 4th International Conference on Wireless Communications, Networking and Mobile Computing, Oct. 12-14, IEEE Xplore Press, Dalian, pp: 1-4. DOI: 10.1109/WiCom.2008.578

Jacquet, P., P. Muhlethaler, T. Clausen, A. Laouiti, A. Qayyum and L. Viennot, 2001. Optimized link state routing protocol for ad hoc networks. Proceedings of the 5th IEEE Multi Topic Conference, Aug. 7-7, Springer, USA., pp: 62-68. DOI: 10.1109/INMIC.2001.995315

Johnson, D.B. and D.A. Maltz, 1996. Dynamic Source Routing in Ad Hoc Wireless Networks. In: Mobile Computing, Imelinsky, T. and H. Korth (Eds.)., Chapter 5, Kluwer Academic Publishers, Norwell, MA, USA., ISBN: 0792396979, pp: 153-181.
Ni, S., Y. Tseng, Y. Chen and J. Sheu, 1999. The broadcast storm problem in a mobile ad hoc networks. Proceeding of the ACM/IEEE International Conference on Mobile Computing and Networking, Aug. 15-19, AMC Press, Seattle, Washington, United States, pp: 151-162. DOI: 10.1145/313451.313525

Perkins, C., E. M. Royer and S.R. Das, 2003. Ad Hoc On-demand Distance Vector (AODV) routing. Internet-Draft, draft-ietf-manet-aodv-13.txt (Work in progress). http://tools.ietf.org/id/draft-ietfmanet-aodv-13.txt

Royer, E., P. Melliar-Smith and L. Moser, 2001. An analysis of the optimum node density for ad hoc mobile networks. Proceedings of the IEEE International Conference on Communications, (ICC'01), Helsinki, Finland, pp: 857-861. DOI: 10.1109/ICC.2001.937360

Siddique, A., A.M. Hanashi, I. Awan and M. Woodward, 2007. Performance evaluation of dynamic probabilistic flooding using local density information in MANETs. Network-Based Inform. Syst., 4658: 288-297. DOI: 10.1007/978-3-54074573-0

Stojmenovic, I., M. Seddigh, and J. Zunic, 2002. Dominating sets and neighbor elimination-based broadcasting algorithms in wireless networks. IEEE Trans. Parall. Distribut. Syst., 13: 14-25. DOI: $10.1109 / 71.980024$

Tseng, Y.C., S.Y. Ni and E.Y. Shih, 2003. Adaptive approaches to relieving broadcast storms in a wireless multihop ad hoc networks. IEEE Trans. Compt., 52: 545-557. DOI: 10.1109/TC.2003.1197122

Wu, J. and W. Lou, 2003. Forward-node-set-based broadcast in clustered mobile ad hoc networks. Wireless Commun. Mobile Comput., 3: 155-173. DOI: 10.1002/wcm.109

Yongsheng, F., W. Xinyu, and L. Shanping. 2008. Performance comparison and analysis of routing strategies in mobile ad hoc networks. Proceedings of the International Conference on Computer Science and Software Engineering, Dec. 12-4, IEEE Xplore Press, Wuhan, Hubei, pp: 505-510. DOI: 10.1109/CSSE.2008.799

Zhang, Q. and D.P. Agrawal, 2005. Dynamic probabilistic broadcasting in MANETs. J. Parall. Distribut. Compt., 65: 220-233. DOI: 10.1016/j.jpdc.2004.09.006 